\title{
La politique du patrimoine en Espagne
}

\author{
Emmanuel Négrier
}

Pour Culture et Musées

\author{
Décembre 2005
}

\begin{abstract}
Résumé :
Cet article propose une analyse des politiques espagnoles en matière de patrimoine culturel. Celles-ci sont fondées sur une double dimension institutionnelle, à la fois centrale et locale, dans un pays fortement décentralisé. La première partie parcourt les différentes étapes de l'institutionnalisation de ces politiques, depuis les Lumières jusqu'à la transition démocratique. Celle-ci, qui s'est fortement appuyée sur le transfert de compétences aux communautés autonomes, a directement touché à l'économie des politiques patrimoniales, qui ont été l'un des tous premiers domaines décentralisés. Mais les effets de différenciation auxquels conduit ce nouveau régime d'action publique sont loin de ne soulever que des enjeux techniques ou juridiques. Dans l'Espagne contemporaine, les politiques du patrimoine sont aussi au cœur de l'identité nationale, de son rapport au passé. Celui, lointain, des fondations de l'unité est traversé de tensions réactivées par l'élaboration même des stratégies patrimoniales autonomiques. Le passé, plus proche, lié à l'héritage franquiste ne fait pas moins débat d'un point de vue patrimonial. Ces questions de cadre réglementaire et financier comme de substance mémorielle et de classement s'inscrivent enfin dans un modèle de politique culturelle largement inachevé.
\end{abstract}

\section{Abstract :}

This article proposes an analysis of Spanish cultural heritage policies. These one are based on a double institutional dimension, simultaneously local and national, in a country which is deeply decentralized. The first part scans the distinct historical steps of the institutionalization of such policies, from the Enlightenment until the democratic transition of the seventies. This transition has clearly opted for an important dynamics of devolution of powers and competences to the Autonomous Communities (regional authorities). It has directly affected the economy of heritage policies, one on the first sector for which such a devolution had been implemented. The impact of this devolution deals with much more than only technical or judicial aspects of the differentiation. Within contemporary Spain, the heritage policies are in the throes of the problem of national identity, and its relationship to the pasts. The first and faraway past, i.e. the foundations of the national Unitarianism, is actually beated up by tensions which are related to the implementation of specific heritage policy strategies, within each autonomous community. The closer past, i.e. the Franco, induces nowadays a new and crucial debate, from an heritage perspective. At least, the article shows how such institutional and technical dimensions of all heritage policies are closely related to a model of cultural policy which remains incomplete.

Introduction

Les politiques culturelles témoignent, à plusieurs égards, du laboratoire européen de la diversité (Genieys 2002) que représente aujourd'hui l'Espagne «multi-nationale» (Linz 
1993). En leur sein, le patrimoine est un domaine spécifique à plusieurs égards, même si certains traits le rapprochent de la situation française. Ainsi, depuis les années 1980, on a pu constater que les gouvernements conservateurs continuaient de lui consacrer un effort budgétaire proportionnellement plus important qu'au cours des législatures socialistes. Mais la politique du patrimoine a dû très tôt tenir compte de sa double nature : une politique centrale dont l'héritage franquiste ne fut pas le moindre des problèmes; une politique décentralisée qui pose, comme pour les autres secteurs, de redoutables enjeux de coordination. C'est ce que nous examinerons dans un premier temps en parcourant les différentes étapes de l'institutionnalisation d'une politique du patrimoine, depuis les Lumières jusqu'à la transition démocratique. Celle-ci, qui s'est fortement appuyée sur le transfert de compétences aux communautés autonomes, a directement touché à l'économie des politiques patrimoniales, qui ont été l'un des tous premiers domaines décentralisés. Mais les effets de différenciation auxquels conduit ce nouveau régime d'action publique sont loin de ne soulever que des enjeux techniques ou juridiques. Dans l'Espagne contemporaine, les politiques du patrimoine sont aussi au cœur de l'identité nationale, de son rapport au passé. Celui, lointain, des fondations de l'unité est traversé de tensions réactivées par l'élaboration même des stratégies patrimoniales autonomiques. Le passé, plus proche, lié à l'héritage franquiste ne fait pas moins débat d'un point de vue patrimonial. Ces questions de cadre réglementaire et financier comme de substance mémorielle et de classement s'inscrivent enfin dans un modèle de politique culturelle largement inachevé.

\section{Lumières, Ombre et diversité}

La politique du patrimoine s'inscrit dès ses premières étapes dans un double processus de construction étatique et d'imitation française. Au XVIII ${ }^{\text {ème }}$ siècle, Felipe V fonde la Bibliothèque Nationale. Sous l'impulsion de son fils, Carlos III, sont créés l'Académie royale des Beaux-Arts et le Musée du Prado, initialement conçu comme cabinet d'histoire naturelle et académie des sciences. Ces dernières institutions, financées par la confiscation des biens des jésuites consécutive à leur expulsion, donneront naissance à la première administration du patrimoine. Constituée alors d'inspecteurs des beaux-arts, elle incarne une volonté de mise à distance de l'influence des ecclésiastiques sur les biens culturels et leur mise à disposition du public (Fernandez-Prado 1991). Mais elle deviendra très vite, au cours du XIX ${ }^{\text {ème }}$ siècle, le siège d'un conservatisme artistique et culturel que contesteront, d'une part, les villes et provinces, et les milieux bourgeois éclairés, qui fonderont, dans les cités espagnoles, les Ateneos, cercles d'animation et de diffusion artistique et culturelle. Par ailleurs, la 
désamortización (confiscation des biens des ordres religieux suite à la suppression de ces derniers en 1835) conduira à la vente d'un très grand volume de biens artistiques privés et l'extension considérable des besoins de muséification. La loi Moyano (1857) institue les Commissions scientifiques et artistiques provinciales, qui deviendront ensuite les Commissions aux Monuments Historiques et Artistiques. Elle fonde également le corps des archivistes et bibliothécaires (Bonet 1999). À l'aube de la guerre civile, la notion de patrimoine culturel s'est donc fortement étendue et diffusée sur l'ensemble du territoire, de façon certes inégale. Elle s'est également enrichie, au cours de la période républicaine (1931), dont la Constitution (articles 45 et 48 ) fait du service culturel une attribution essentielle de l'État et consacre l'intérêt public national du patrimoine. La loi de protection du trésor artistique (1931) définit une politique en cette matière, autour des principes de protection, de d'ouverture au public et de contrôle de la commercialisation. L'extension de la reconnaissance publique du patrimoine doit cependant être nuancée par l'exiguïté des moyens que l'État lui consacre effectivement et, surtout, par l'instabilité politique qui la prive, notamment pendant le bienio negro (la dictature Primo de Rivera 1933-1935), de toute perspective institutionnelle. L'essentiel du cadre réglementaire du patrimoine sera donc mis en œuvre, dans des conditions très spécifique, par le régime franquiste.

La politique culturelle franquiste se démarque, en Europe du Sud, de celle suivie par l'Italie fasciste ou la dictature salazariste portugaise. Vis-à-vis de la première, elle ne prétend pas construire une esthétique de régime telle que le Minculpop en a forgé le projet. Elle se limite à la mise en œuvre de politiques culturelles basées, comme dans tout régime autoritaire, sur une utilisation intensive de la censure et la glorification d'un patrimoine et d'un folklore artificiellement «hispanisés ». Vis-à-vis de la seconde, la politique culturelle franquiste ne démontre pas la même défiance de principe à l'égard de l'intervention culturelle, en inscrivant au contraire les politiques de Téléclubs ${ }^{1}$ ou de Casas de Cultura, dans les années 1960, dans le droit et paradoxal fil d'André Malraux. Il y a dans le franquisme l'expression d'une volonté d'intervention culturelle qui se veut, comme dans la seconde phase de la Grèce des colonels, un symbole d'ouverture à l'occidentale, tout en fonctionnant au quotidien sur la répression

\footnotetext{
${ }^{1}$ Les téléclubs espagnols résultèrent d'une décision de la Direction Générale de l'Information et du Tourisme, le 6 avril 1964, de diffuser sur l'ensemble du territoire un modèle collectif de réception de la télévision, dont l'objectif initial était double : permettre la réception de ce média dans des territoires enclavés, ruraux et aux ressources faibles, et encadrer cette réception par le truchement d'animateurs très fortement liés au régime franquiste. Le premier aspect ne se traduisit cependant pas par une diffusion très large du réseau national de téléclubs, qui s'avéra obsolète au gré de l'équipement individuel des ménages. Le second fut, dans la pratique bien moins idéologiquement performant qu'attendu. Selon Chus Cantero (2006), la mise en œuvre des téléclubs donna lieu à beaucoup plus de discussions pluralistes localisées qu'à un endoctrinement unilatéral.
} 
des libertés de création et d'expression des identités sub-nationales ${ }^{2}$. Dès lors, la politique patrimoniale du franquisme est le résultat d'utilisation de trois types d'instruments. Le premier est la maîtrise d'un large secteur patrimonial (monuments et musées, bibliothèques, archives) qui s'appuie notamment sur le contrôle des académies, regroupées au sein d'un Institut d'Espagne, dès avant la fin de la guerre civile, afin d'en obtenir le soutien. Le deuxième est l'hispanisation de l'identité de référence des éléments patrimoniaux, avec une tendance à la folklorisation des pratiques culturelles légitimes et au rejet des traditions particularistes. Le troisième est le recours à la valorisation touristique et commerciale de toute une série de monuments, réhabilités et transformés en hôtels et restaurants de luxe : les paradores. Cette politique exprime plusieurs finalités, et notamment la nécessité d'un financement de la restauration du patrimoine qui ne trouve, dans un pays exsangue, que peu de ressources à ses ambitions; et la mise en exergue d'un réseau monumental ouvert à la clientèle touristique occidentale aisée, censée réinsérer l'Espagne dans le concert libéral européen.

Le legs de cette longue période autoritaire est, pour le patrimoine, celui d'une embarrassante priorité. La transition démocratique s'opère en préservant l'essentiel de l'héritage matériel du patrimoine, mais en en transférant radicalement le mode de gestion politique. Elle a fait du secteur culturel un domaine fortement décentralisé. L'État a certes conservé certaines compétences exclusives, au titre des articles 149-1 et 149-2 de la Constitution de 1978, qui se rapproche d'une logique fédérale : les normes visant les droits d'auteurs, les relations culturelles extérieures, la protection des biens patrimoniaux ; une mission de faciliter la communication culturelle entre les communautés autonomes (Prieto de Pedro 1993). Ce maintien d'une compétence étatique a justifié la présence d'un ministère de la culture qui, en fonction des différentes périodes, a connu une existence autonome, dans la phase socialiste 1982-1996 (Rubio 2003) ou a été intégré à un plus vaste ministère de l’Éducation, de la culture et des sports, comme dans les gouvernements du Parti Populaire, de 1996 à 2004 (Négrier 2004). Il a retrouvé son autonomie depuis le retour des socialistes au pouvoir, en mars 2004.

À la conception centraliste et univoque succède une décentralisation d'une très large partie des compétences, monuments et capacités d'action. Cette décentralisation s'appuie sur des transferts de ressources fiscales, sur la capacité législative dont, dans ce domaine comme dans

\footnotetext{
${ }^{2}$ À ceci près que l'identité «patrimoniale » de la culture vivante est, pour le franquisme, un folklore massivement andalou (flamenco, corrida, etc.).
} 
d'autres, les Communautés autonomes bénéficient. Il en découle une pluralisation des modèles d'action patrimoniale et, surtout, des références fondant les principes de classement. Chacune des communautés, à commencer par celles d'entre elles qui sont dites « historiques » (Catalogne, Pays-Basque, Galice) entend récupérer non seulement les ressources d'action mais une autonomie dans la manière de considérer ce qui relève de sa propre construction identitaire. Cette circonstance n'est pas sans peser sur les relations entre niveaux d'action. En effet, d'une part, l'Etat conserve des attributions et capacités d'action. D'autre part, les communes, qui ne sont pas attributaires de compétences décentralisées, sont pourtant des acteurs de premier plan dans l'action publique patrimoniale concrète. Cette complexité se redouble de l'étendue du domaine à couvrir désormais.

La décentralisation des compétences culturelles impose, en matière de patrimoine, l'adoption d'une législation nouvelle, qui remplace celle de 1931, toujours en vigueur officiellement. La loi sur le Patrimoine Historique Espagnol du 29 juin 1985 prend acte de la nouvelle répartition des attributions. L'État conserve une responsabilité en matière d'exportation, de spoliation et de gestion des monuments et institutions de statut étatique, peu nombreuses. Les Communautés Autonomes se voient attribuer tout ce qui relève de l'intérêt pour la Communauté Autonome». Ce transfert s'accompagne de celui des services et fait, en pratique, des Communautés le véritable centre de gravité de la politique du patrimoine. Celles-ci ont engagé un processus législatif selon deux types : approbation d'une loi globale sur leur patrimoine, ou processus par étape selon chacun des domaines (archives, bibliothèques, monuments...). Aujourd'hui, seules trois Communautés n'ont pas encore adopté de loi générale, et celles qui l'ont faite se sont largement inspirées du modèle national pour l'adapter à leur réalité territoriale (Rausell 2006).

Cet isomorphisme législatif entre l'État et les autonomies a sans doute contribué à faire que le patrimoine donne lieu, contrairement à tous les autres secteurs culturels, à une certaine coordination entre ces deux pôles. Le fait que chacun ait conservé des attributions précises les rend certes interdépendants. En outre, certains dispositifs permettent d'assurer cette coordination de façon régulière, comme le Conseil du Patrimoine Historique, l'harmonisation des procédures de déclaration des Biens d'Intérêt Culturel, l'Inventaire Général des Biens mobiliers, les Systèmes Espagnols des Archives, des Bibliothèques et des Musées, etc. Cependant, cette coordination et ce rapprochement par les outils et par les standards professionnels ne font pas obstacle à une réelle diversité dans la manière dont chaque 
autonomie construit « son » identité patrimoniale. Ils n'aboutissent pas davantage à un champ consensuel sur ce qu'est une politique patrimoniale légitime ou concertée.

Tableau : le patrimoine dans les dépenses culturelles de l'Etat et des Communautés autonomes (19912000) (en millions d'euros)

\begin{tabular}{|c|c|c|c|c|c|c|c|c|c|}
\hline & & 1991 & 1993 & 1994 & 1996 & 1997 & 1998 & 1999 & 2000 \\
\hline $\begin{array}{l}\text { Communautés } \\
\text { autonomes }\end{array}$ & Budget culturel total & 590,7 & 679,4 & 681,9 & 670,7 & 742,3 & 802,0 & 865,0 & 945,4 \\
\hline & Patrimoine & 124,0 & 143,4 & 158,3 & 201,9 & 210,4 & 247,4 & 253,4 & 298,5 \\
\hline & $\%$ sur le total & $21 \%$ & $21 \%$ & $23 \%$ & $30 \%$ & $28 \%$ & $31 \%$ & $29 \%$ & $32 \%$ \\
\hline Etat & Budget culturel total & 435,9 & 438,7 & 667,3 & 635,8 & 535,1 & 542,4 & 583,4 & 716,0 \\
\hline & Patrimoine & 87,0 & 90,1 & 84,3 & 93,4 & 92,9 & 97,6 & 109,8 & 127,5 \\
\hline & $\%$ sur le total & $20 \%$ & $21 \%$ & $13 \%$ & $15 \%$ & $17 \%$ & $18 \%$ & $19 \%$ & $18 \%$ \\
\hline
\end{tabular}

Source : « Las cifras de la cultura en España », Ministère de l’Éducation et de la Culture, Madrid 2002

La dynamique de décentralisation est claire. Non seulement l'État a largement été dépassé par le développement des politiques autonomiques, mais encore faut-il remarquer que le secteur du patrimoine est d'une importance croissante dans le budget de ces dernières (de $21 \%$ à $32 \%$ des budgets) tandis qu'il a tendance à régresser dans le budget de l'Etat, mais de façon variable au gré des périodes et législatures. Ainsi, et de façon assez récurrente en Europe du sud, le poids du patrimoine dans les budgets publics tend à être plus important lorsque le parti conservateur est au pouvoir. Cette circonstance s'explique notamment par le fait que le patrimoine espagnol, comme l'italien, accorde une très large place aux édifices catholiques, et donc à une église qui constitue une part importante de la sociologie de ces partis (Dubois et Négrier 1999).

\section{Patrimoines, mémoires et politique}

L'État et les Communautés autonomes, auxquels il convient d'ajouter les municipalités, ont à affronter la gestion d'un patrimoine qui, à l'instar des pays méditerranéens, est extrêmement riche, menacé autant que symbolique, aussi coûteux que susceptible de valorisation commerciale. Depuis plus d'une décennie, une série de plans pluriannuels ont été conçus à l'échelle nationale, comme le Plan des Cathédrales ou le Plan de Réhabilitation des Théâtres. 
Leur objectif est d'une part de maintenir une vocation de l'Etat central à intervenir dans ce domaine, selon une double logique d'aménagement du territoire et de marquage symbolique sur des monuments particuliers. L'autre finalité de cette intervention, plus triviale, est de mettre fin à une carence d'intervention publique dans la période franquiste, laquelle n'a pas réellement mis les moyens publics à l'appui de son évocation très spécifique de l'hispanité patrimoniale. Enfin, la définition d'une action planificatrice, dont l'ampleur reste cependant limitée, permet de soutenir la réalité des compétences « constitutives » (régulation juridique et coordination technique) qu'il détient par ailleurs.

À la multiplicité des interventions des pouvoirs publics de différents niveaux (Etat, Communautés Autonomes, Provinces et communes), s'ajoute l'existence d'instruments placés sous la responsabilité de plusieurs types d'autorité sectorielle. Par exemple, une partie du financement d'opérations de rénovation du patrimoine passe par la gestion du $1 \%$ culturel, géré par le Ministère de l'Équipement, en coordination avec le Ministère de l'Education, de la Culture et des Sports. Celui-ci est, comme en France, centralisé sur les opérations d'envergure nationale, et adapté dans le cadre de chaque législation autonomique (Arroyo Yanes 2004).

Les enjeux patrimoniaux contemporains renvoient à plusieurs niveaux de questionnement. Le premier est de nature politico-administrative, au sein des politiques culturelles. Le deuxième a trait à l'évolution des pratiques de valorisation. Le troisième concerne enfin le rapport entre la cohérence professionnelle et juridique de cette politique publique et la diversité dont la notion de patrimoine tend aujourd'hui à témoigner en pratique.

En tant que sous-secteur des politiques culturelles, le patrimoine occupe un rôle prééminent. Première compétence culturelle réellement transférée, c'est sur elle que les transformations professionnelles et les nouvelles vocations autonomiques se sont exercées. Le poids du patrimoine en termes de dépense publique fait aujourd'hui l'objet d'un débat sur le point de savoir s'il doit demeurer prioritaire par rapport à d'autres sous-secteurs tels que les arts de la scène, la création en général (Lacombe 2004), les industries culturelles traditionnelles ou nouvelles (Cubeles 2006). Ce débat, dans les communautés autonomes, a ceci de spécifique que le patrimoine y incarne encore la récupération d'une identité qui, sous le franquisme, avait fait l'objet d'une large occultation. Il est donc, notamment pour les élites autonomistes modérées, à l'idéologie plutôt conservatrice (exemple : Convergencia y Union en Catalogne), entourée d'une valeur symbolique très forte. 
La forte présence de l'enjeu patrimonial dans les budgets autonomiques soulève ensuite tous les enjeux liés à la modernisation de ces politiques, au-delà des seuls aspects professionnels de la conservation, de la protection et de la mise en valeur. Ainsi, on peut indiquer trois premiers enjeux liés à la modernisation, déjà engagée, des politiques menées aux échelles nationale, autonomique et locale. Le premier est le développement de l'animation du patrimoine auprès des populations, par la mise en œuvre de plans d'interprétation du patrimoine, comme garantie de son usage social, et de l'appropriation du patrimoine comme élément clef de l'identité locale; ces enjeux concernent à la fois le diffusion de la connaissance du patrimoine auprès des populations concernées en général, mais aussi auprès de populations plus ciblées : les publics scolaires, les professions touristiques, les promoteurs du développement local. C'est l'enjeu de l'usage social du patrimoine.

Le deuxième enjeu est l'articulation entre les objectifs intrinsèques de conservation et protection et les finalités extrinsèques de valorisation économique et touristique. C'est l'enjeu patrimonial du développement local. Il s'agit à la fois de permettre la mise en valeur des éléments du patrimoine et de lutter contre les effets potentiellement négatifs de l'usage commercial ou touristique «basique » des monuments et objets patrimoniaux (conflits sur l'usage, dégradation des bâtiments, production d'une fausse culture touristique...etc). Il s'agit aussi de rendre plus systématique l'utilisation culturelle et artistique des lieux patrimoniaux (organisation de festivals, de spectacles, de résidences d'artistes, etc...). Enfin, le dernier défi est la production d'une offre territoriale cohérente autour de la notion de réseau patrimonial (musées, monuments, objets), qui permette l'amélioration du partenariat entre lieux, institutions et pouvoirs publics. C'est l'enjeu de la subsidiarité et de la concertation des politiques patrimoniales.

Trois thématiques sont enfin en rapport direct avec les débats contemporains plus larges au sujet du patrimoine espagnol, de sa diversité et des limites de son objet. Il s'agit d'abord de l'extension des politiques patrimoniales dans des domaines nouveaux comme le patrimoine industriel, encore très largement sous-valorisé. On entend par là les pratiques de classement qui touchent à des éléments bâtis : les fabriques dont la préservation est assurée par leur réutilisation artistique ou culturelle, comme dans le cas de la Farinera à Barcelone, de la Casa Incendida à Madrid ou de l'Institut Andalou du Patrimoine Historique à Séville. Mais on entend aussi l'ensemble des processus qui ont accompagné cette industrialisation et qui touche davantage aux mémoires sociales, comme l'illustre le Musée de l'immigration projeté à Barcelone, qui vise à restituer l'épopée de toutes les immigrations industrielles des 
murciens, andalous et autres manchegos qui sont venus grossir les rangs prolétaires de la métropole barcelonaise au cours du $\mathrm{XX}^{\mathrm{ème}}$ siècle. Parallèlement, d'autres projets concernent un travail de mémoire (photographique essentiellement) de l'exode rural, ou de l'ethnologie villageoise, qu'illustre un projet d'institut de l'image actuellement en cours dans la Communauté autonome d'Extrémadure.

Mais le cas le plus complexe vise le travail de mémoire contemporaine. Celle-ci touche à plusieurs objets qui revisitent la mémoire des exils et de la guerre civile. Il a fallu attendre 2003 pour qu'une première exposition photographique ait lieu, à Madrid, sur l'exil des Républicains contraints de fuir l'Espagne franquiste. Cette thématique, qui est encore sujet à une vive sensibilité, occupe le devant de la scène politique à l'occasion de multiples films, expositions photographiques, et excavations (notamment des charniers où furent enfouis sans sépulture des combattants de la guerre civile). On est, dans ce domaine à la charnière entre mémoire historique et patrimoine culturel, en train de revenir sur les principes, politiquement explicites à l'heure de la transition démocratique des années 1970, de rejet de tout examen à vif des responsabilités et crimes liés à la guerre civile et aux décennies de franquisme. La meilleure preuve de la sensibilité encore exacerbée de cet enjeu est la présence, en Espagne, de nombreuses rues portant encore le nom de dignitaires du régime franquiste, dont celui du généralissime, et de statues du caudillo encore présentes sur certaines places publiques. La velléité de démonter les statues et de rebaptiser les rues conduit désormais à la réactivation de groupes nostalgiques des années noires. À sa manière, elle pose aujourd'hui la question embarrassante de savoir si le franquisme et sa monumentalité peuvent ou non faire l'objet d'une patrimonialisation et, si oui, de quelle manière.

Enfin, attestant du fait que la répartition des compétences issues de la transition démocratique et de la loi sur le patrimoine historique n'a pas tout réglé, la politique espagnole du patrimoine doit affronter d'autres luttes de classement à propos d'un passé plus lointain fondé sur une identité plus proche, autonomique. Deux cas, qui concernent tous deux la Catalogne, témoignent de cet enjeu. Le premier est une anecdote édifiante ; le second un conflit de longue date.

À Barcelone, le Marché du Born est une structure métallique de style Baltard construit en 1874. En 1971, le déplacement définitif, vers la périphérie, de ce marché pose la question de la démolition du bâtiment. Parallèlement, l'Etat espagnol qui, en vertu d'une loi datant de la fin du XIX ${ }^{\text {ème }}$ siècle, toujours en vigueur, s'engageait à construire un bibliothèque centrale par province, n'avait toujours pas rempli ses obligations pour la Province de Barcelone. L'accord se fit sur la requalification de ce site, sous l'angle d'une requalification-préservation d'un 
patrimoine industrialo-marchand. Mais lorsque les excavations débutèrent, on réalisa qu'une ville entière resurgissait, à moins de deux mètres de profondeur. Le Marché du Born recouvrait en réalité les restes d'un ancien quartier médiéval bombardé par les troupes Bourbon, lors du siège de Barcelone, en 1714, le 11 septembre, désormais date de la fête nationale catalane, car jour de deuil de son ultime espoir d'indépendance. Cette découverte entraîna une polémique sur la préservation d'un tel patrimoine, et sur le bien fondé d'un financement, par l'Etat espagnol, d'un lieu de mémoire dédié à la souveraineté perdue de la Catalogne... (L’Avenç 2002). Le gouvernement, alors dirigé par le Parti Populaire, ne souhaita pas s'engager dans ce « piège » et négocia un autre emplacement plus « neutre ».

À Salamanque sont regroupées les Archives historiques de l'État espagnol et de ses étapes successives. Actuellement propriété de l'Etat espagnol, elles concernent la plupart des Communautés, en particulier. À l'occasion de la guerre civile, en 1939, les Archives de la Généralité furent dérobées par les troupes franquistes, puis regroupées à Salamanque ${ }^{3}$. Depuis la transition démocratique, le retour de ces archives agite une polémique entre élites « espagnolistes » favorable à leur maintien à Salamanque, et courants catalans, désireux de leur retour à Barcelone ${ }^{4}$. Le dossier, qui n'avait guère progressé durant les législatures socialistes, a été explicitement enterré par les gouvernements Aznar, dont l'orientation « néoespagnoliste » était régulièrement dénoncée. Il a été récemment examiné, par le biais d'un rapport d'experts, par le nouveau ministère socialiste, et le retour a été entériné par une loi en 2005. La question de la destination de ce patrimoine rejoint d'autres polémiques, comme celles liées aux archives de la Couronne d'Aragon ${ }^{5}$, déposées à Barcelone, mais gérées par l'État espagnol. La Communauté autonome d'Aragon, de même que celle de Valence estiment désormais qu'elles ont un droit égal à celui de la Catalogne à en être les titulaires légitimes. D'autres controverses concernent des patrimoines muséaux qui sont à la frontière entre deux communautés autonomes, comme l’illustre le cas du musée épiscopal de Lleida (Lerida, ouest de la Catalogne). Celui-ci possède des pièces correspondant aux limites du

\footnotetext{
${ }^{3}$ Ces archives, parmi lesquelles on comptait les registres des partis et syndicats, ont été utilisées par le franquisme pour la politique de répression systématique des premières années du régime. Elles comportent aussi des pièces plus personnelles, comme des lettres de prison.

${ }^{4}$ Il faut cependant noter que le premier élu à avoir réclamé ce retour des archives fut, en 1982, un député de l'Alliance Populaire de Catalogne (soit le parti le plus espagnoliste, qui est l'ancêtre du Parti Populaire). Ce sont les professionnels du patrimoine, une corporation alors encore dominée par des forces ultra-conservatrices, qui avaient fait front.

${ }^{5}$ La couronne d'Aragon, au plus fort de son histoire, en 1479, regroupait les royaumes d'Aragon, de Majorque, de Valence, de Sardaigne et le comté de Barcelone. L'exhumation, pour l'État espagnol, d'un tel spectre atteint jusqu'aux fondements de sa propre existence.
} 
diocèse, qui déborde la frontière catalane et concerne aussi la partie orientale de l'Aragon, catalanophone. L'Aragon exige, en parallèle du retour des Archives de Salamanque, celui des archives aragonaises que ce musée détient.

Ces différents traits de l'action patrimoniale en Espagne et, on l'aura compris, non exclusivement « espagnole » montrent à l'évidence que, au-delà de l'acquis professionnel, budgétaire et juridique d'un secteur à pluralité de niveaux d'intervention, la question patrimoniale est intrinsèquement politique. Les variations qui se rapportent à la manière dont chacune des communautés autonomes revisite son propre passé mettent en question la construction identitaire espagnole. D'autres pratiques renvoient plus loin encore, comme les fêtes des Chrétiens et des Maures, où se rejouent les luttes historiques de la Reconquista, notamment en Andalousie. Elles heurtent aussi les phénomènes plus récents de sourde hostilité à l'égard de l'immigration subsaharienne et maghrébine, qui ont défrayé la chronique ces dernières années. La nature de ces enjeux rend assez subtile la dialectique entre la cohérence (de gestion, de compétence technique et professionnelle) et la diversité (des mémoires, des savoir-faire, des stratégies identitaires).

\section{Le modèle de politique culturelle}

Sans remettre en cause l'architecture constitutionnelle de la dialectique entre l'unité et la diversité du modèle espagnol de politique culturelle, il existe à l'heure actuelle un double débat sur le modèle de politique publique qui concerne la culture.

Le premier débat concerne la nature et l'extension de la décentralisation culturelle. La situation actuelle est, principalement, liée au fait que l'Etat a concédé d'importantes compétences aux Communautés autonomes. Par contre, ces dernières n'ont pas relayé l'esprit de subsidiarité en direction des municipalités, et des villes en particulier (Zallo 2001). Or on sait que ce sont les villes qui, aujourd'hui (comme dans la totalité des autres pays européens), assument la part majeure du financement culturel et des nouveaux enjeux de ces politiques (démocratisation, démocratie et développement culturels). L'hypothèse d'une nouvelle étape de décentralisation culturelle est donc sur l'agenda, même si elle ne passe pas nécessairement par l'octroi de compétences précises et obligatoires en matière culturelle, à destination des villes (Font 2000). Elle renvoie à trois outils distincts. 
Le premier est la confortation des ressources des villes, en termes d'action publique en général (ressources fiscales, capacités budgétaires). Les villes espagnoles, qui concentrent une part croissante de la population, souffrent d'un handicap général dans ce domaine (Lopez de Aguileta 2000).

Le deuxième outil vise la constitution d'institutions de coopération au-delà du strict niveau municipal, et concerne la gestion métropolitaine des équipements, projets et politiques culturelles. La plupart des villes assument de plus en plus des charges centrales au profit d'une population qui s'étend, autour des villes, à des ensembles métropolitains de plus en plus larges (Interart's 2002). En dehors peut-être de la Communauté autonome de Madrid (qui fonctionne à la fois comme une Communauté Urbaine et comme une région institutionnelle), la plupart des agglomérations espagnoles restent aujourd'hui sans réelle institution à la mesure des enjeux publics et des problèmes d'action publique.

Le troisième outil consiste dans le développement de dispositifs de partenariat entre les Communautés Autonomes, les Villes et, selon les territoires spécifiques, les provinces, lorsqu'elles ont conservé, comme à Barcelone, des compétences en matière culturelle. Ces dispositifs existent aujourd'hui de façon ponctuelle, pour un établissement ou un projet particuliers (au travers des consorcios et mancomunidades). Ces partenariats, au-delà des acteurs publics, concernent aussi l'implication de la société civile (les entreprises privées, les associations) dans la mise en œuvre des finalités de politique culturelle.

Le second élément de débat se rapporte à l'organisation ministérielle en tant que telle. Aujourd'hui, le ministère de la culture demeure marqué par un héritage centraliste dans un contexte pourtant fortement décentralisé. Son organisation interne reflète l'esprit d'un département généraliste, fondé sur des directions thématiques et sectorielles, alors que la finalité de son intervention est devenue, à travers la décentralisation, plus transversale. On peut donc penser que cette organisation est aujourd'hui en décalage avec la réalité des missions du ministère.

\section{Conclusion}

Dans cet article, nous avons voulu souligner la nature de l'expérience espagnole de politique patrimoniale, en alternant les éléments de spécificité et les convergences qui en marquent l'évolution. On peut schématiquement suggérer trois temps distincts de comparaison. Les origines historiques de l'action patrimoniale ne sont pas si différentes en Espagne de ce 
qu'elles furent dans beaucoup de pays, et notamment la France, bien que certaines circonstances (l'expulsion des jésuites) demeurent particulières. Les aspects les plus spécifiques sont en même temps ceux liés à l'histoire moderne, et en particulier à la longue période du franquisme. Celle-ci, qui a mis l'accent sur un patrimoine historique et vernaculaire artificiellement unifié, a marqué le régime public du patrimoine d'une manière telle qu'elle la rend difficilement comparable avec d'autres pays européens. Le niveau de comparaison le plus intéressant, mais qui suppose autant de nuances que d'homologies, est celui des pays d'Europe du sud, qui ont vécu, de façon plus ou moins intense et longue, de semblables régimes. Enfin, la période la plus contemporaine des politiques du patrimoine, celles qui correspondent à l'Espagne postérieure à la transition démocratique, montrent le retour à des problématiques que partagent la plupart des politiques actuelles du patrimoine, depuis les fonctions classiques de protection et de mise en valeur jusqu'à la valorisation commerciale, l'animation pédagogique et la diversification des références esthétiques. Dans la phase actuelle, la comparaison entre l'Espagne et la France, par exemple, ne peut éviter la question institutionnelle, et notamment la dynamique différente de relations et de compétence entre l'Etat central et les différents niveaux de collectivité territoriale. Cependant, et compte tenu des évolutions sensibles en France à ce sujet, on peut se demander combien de temps cette différence de modèle perdurera et en quoi l'Espagne pourrait s'avérer un laboratoire d'une nouvelle organisation territoriale du patrimoine. On a vu, à ce sujet, qu'il convenait de ne pas idéaliser un système qui, s'il repose sur une large diffusion de la légitimité du secteur dans les politiques décentralisées, n'en comporte pas moins un certain nombre d'angles morts et de contraintes. 


\section{Références citées :}

Arroyo Yanes, Luis Miguel (2004) : «La protección del patrimonio a travès del porcentaje cultural : modelos legales y propuestas de reforma », Periférica n ${ }^{\circ} 4$, pp.39-54

Bonet, Lluis (1999): “La politique culturelle en Espagne : évolution et enjeux ”, Pôle Sud $n^{\circ} 10$, pp.58-74

Cantero, Chus (2006: «Equipamientos culturales de proximidad en España en el siglo XX : los teleclubs », Periférica nº, 2006, pp. 105-126

Cubelles, Xavier (2006) : «Les politiques culturelles et le processus de mondialisation des industries culturelles », in Lluis Bonet et Emmanuel Négrier (dir.), La fin des cultures nationales. Les politiques à l'épreuve de la diversité, à paraître 2006

Dubois, Vincent et Négrier, Emmanuel (1999), «Les politiques culturelles en Europe du Sud », Pôle Sud $\mathrm{n}^{\circ} 10$, pp.3-10

Fernandez Prado, Emiliano (1991), La politica cultural : qué es y para qué sirve, TREA, Gijon

Font, Jordi (2000, dir.), Cultura y poder local. Reflexiones y propuestas desde la Mesa de Concejales de Cultura de los municipios de la provincia de Barcelona, Milenio, Barcelona

Genieys, William (2002 dir.), L’Espagne du politique, Pôle Sud n ${ }^{\circ} 6$

Interart's (2002), La coopération culturelle intercommunale en Catalogne (Espagne), Lombardie (Italie) et Setubal (Portugal), Rapport pour le Ministère de la Culture (DEP-France) Fundacio INTERART'S, Barcelona

L’Avenç - Revista d'Història i Cultura (2002) : « El Born. Ciutat i Memòria », pp.32-81

Lacombe, Robert (2004), Le spectacle vivant en Europe. Modèles d'organisation et politiques de soutien, Paris : Documentation française

Linz, Juan (1993) : «State building and Nation building », European Review Vol.1 n4, pp.355-369, version française : «Construction étatique et construction nationale », Pôle Sud n7, 1997, pp.5-26

Lopez de Aguileta, Inaki (2000), Cultura y ciudad. Manuel de política cultural municipal, TREA, Gijon

Négrier, Emmanuel (2004) : "Actuales cuestiones de debate y desarollo de la política cultural », in Jaime Otero (dir.), La política cultural en España, Madrid: Fundación Elcano, Informe para el Consejo de Europa

Prieto de Pedro, Jesus (1993), Cultura, culturas y constitución, Congreso de los Diputados, CEC, Madrid

Rausell Köster, Pau (2006) La contribution de l'économie aux politiques de protection du patrimoine et de participation des citoyens, in Lluis Bonet et Emmanuel Négrier (dir.), La fin des cultures nationales. Les politiques à l'épreuve de la diversité, à paraître 2006

Rubio Arostegui, Arturo (2003) El Ministerio de Cultura. La política cultural del estado en los gobiernos socialistas: 1982-1996, Univ. Complutense de Madrid, Fac. De ciencias políticas y Sociología, Dept de ciencias política y de administración 1, $486 \mathrm{p}$ 
Zallo, Ramon (2001) : "Políticas culturales territoriales : una experiencia rica pero insuficiente", in Bustamente, Enrique (dir.), Comunicación y cultura en la era digital. Industrias, mercados y diversidad en España, Gedisa Ed., Madrid 